\title{
Conditional Survival of Patients With Nonmetastatic Renal Cell Carcinoma: How Cancer-Specific Mortality Changes After Nephrectomy
}

Carlotta Palumbo, MD,b; Francesco A. Mistretta, MD a,c; Sophie Knipper, MDª, ; Angela Pecoraro, MDa,e; Zhe Tian, PhD Shahrokh F. Shariat, MD, PhD; Fred Saad, MD, FRCSa,g; Claudio Simeone, MD ${ }^{\text {; }}$; Alberto Briganti, MD, PhD h,i; Alessandro Antonelli, MD'; and Pierre I. Karakiewicz, MD ${ }^{\mathrm{a}, \mathrm{g}}$

\begin{abstract}
Background: Conditional survival (CS) may reveal important differences in cancer-specific mortality (CSM) among patients with nonmetastatic renal cell carcinoma (nmRCC). This study assessed CS according to $T$ and $N$ stages in patients treated surgically for $n m R C C$. Patients and Methods: Within the SEER database (2001-2015), all patients with $\mathrm{nmRCC}$ treated with either partial or radical nephrectomy were identified. CSM-free estimates according to $T$ and $\mathrm{N}$ stage and substage groupings (pT1aN0-pT4N0 and pTanyN1) and multivariable Cox regression models with adjustment for Fuhrman grade and histologic subtype were assessed. Results: According to $T$ and $\mathrm{N}$ stage and substage groupings, the following patients were included in the study: 35,966 (46.2\%) with pT1aNO disease; 18,858 (24.2\%) with pT1bN0; 5,977 (7.7\%) with pT2aN0; 2,511 (3.2\%) with pT2bN0; 11,839 (15.2\%) with pT3aN0; 1,037 (1.3\%) with pT3b-cN0; $402(0.5 \%)$ with pT4N0; and 1,302 (1.7\%) with pTanyN1. Conditional CSM-free survival estimates were $98.2 \%$ at 1 year versus $98.0 \%$ at 10 years of event-free follow-up for patients with pT1aN0 disease, relative to baseline. Conversely, pT4N0/pTanyN1 conditional CSMfree survival estimates were $55.8 \%$ at 1 year versus $77.9 \%$ at 8 years of event-free follow-up. Attrition due to mortality was highest in patients with pT4N0/pTanyN1 disease. In multivariable Cox regression analyses, T stage, tumor grade, and histologic subtype represented independent predictors, but no interactions were identified. Conclusions: Tumor stage and its substages represent extremely important determinants of prognosis after lengthy event-free follow-up. The recorded observations have critical importance for physicians regarding patient follow-up and counseling.
\end{abstract}

J Natl Compr Canc Netw 2020;18(1):44-51 doi: $10.6004 /$ jnccn.2019.7350

\footnotetext{
${ }^{a}$ Cancer Prognostics and Health Outcomes Unit, University of Montreal Health Center, Montreal, Quebec, Canada; 'brology Unit, ASST Spedali Civili of Brescia, Department of Medical and Surgical Specialties, Radiological Science and Public Health, University of Brescia, Brescia, Italy; ' Department of Urology, European Institute of Oncology, IRCCS, Milan, Italy; ${ }^{d}$ Martini-Klinik, University Medical Center Hamburg-Eppendorf, Hamburg, Germany; ${ }^{e}$ Department of Urology, San Luigi Gonzaga Hospital, University of Turin, Orbassano, Turin, Italy; fDepartment of Urology, Medical University of Vienna, Vienna, Austria; 'Division of Urology, University of Montreal Hospital Center, Montreal, Quebec, Canada; and h' Division of Experimental Oncology, Unit of Urology, Urological Research Institute, IRCCS Ospedale San Raffaele, and 'Vita-Salute San Raffaele University, Milan, Italy.
}

\section{Background}

Conditional survival (CS) is routinely used in clinical practice. Specifically, clinicians invariably adjust the prognosis of individual patients according to the duration of event-free follow-up. Patients with longer event-free follow-up have a higher probability of remaining disease-free. ${ }^{1}$ However, the magnitude of improved survival is difficult to estimate.

Two studies ${ }^{2,3}$ address cancer-specific CS in patients with nonmetastatic renal cell carcinoma (nmRCC): one is a population-based analysis, ${ }^{2}$ whereas the other is a multi-institutional analysis from European and North American centers of excellence. ${ }^{3}$ Both studies are limited by their relatively historical populations. Moreover, the multi-institutional analysis offers a model for prediction of survival rates after nephrectomy in individual patients, ${ }^{3}$ whereas the population-based analysis provides estimates for the AJCC subgroupings. ${ }^{2}$ Consequently, neither study allows clinicians to assess CS according to $\mathrm{T}$ and $\mathrm{N}$ stage and substage groupings, such as pTlaN0 and pTlbN0. These limitations may be critical with respect to applicability and clinical usefulness.

Based on this unmet need, we investigated cancerspecific CS rates in a contemporary cohort of surgically treated patients with nmRCC, identified within the SEER database (2001-2015). All analyses were stratified according to $\mathrm{T}$ and $\mathrm{N}$ stage and substage groupings. We hypothesized that important reductions in the risk of 5-year cancerspecific mortality (CSM) may be identified with increasing duration of event-free survival (EFS), according to $\mathrm{T}$ and $\mathrm{N}$ stage and substage groupings. Specifically, we postulated that patient subgroups may be identified in whom the risk of CSM is virtually completely eliminated after EFS of several years.

\section{Patients and Methods}

\section{Data Source and Study Population}

Within the SEER database (2001-2015) ${ }^{4}$ we focused on patients aged $\geq 18$ years treated with either partial or 
radical nephrectomy as primary treatment for either pTlaN0-pT4N0 or pTanyN1 nmRCC (ICD-O-3 site codes C64.9). Death was defined according to the SEER mortality code as either CSM (death from RCC) or othercause mortality (death from any other causes). Exclusion criteria consisted of metastatic RCC; unavailable information on $\mathrm{T}$ and $\mathrm{N}$ stage, histology, and Fuhrman grade; diagnosis only on autopsy or death certificate only; and missing follow-up data.

\section{Statistical Analyses}

Our analyses relied on 2 analytical steps. First, conditional 5-year CSM-free rate was quantified in surgically treated patients with nmRCC, according to $\mathrm{T}$ and $\mathrm{N}$ stage and substage groupings (pTlaNo vs pT1bN0 vs pT2aN0 vs pT2bN0 vs pT3aN0 vs pT3b-cN0 vs pT4N0/pTanyN1). CS methodology was applied, as previously reported., Specifically, CS was calculated as the probability of survival for $x$ additional years, given $y$ years of accumulated survival. EFS time points used in the CS models consisted of from 1 year up to 10 years after surgery. Second, separate multivariable Cox regression (MCR) models predicting CSM were fitted to examine the possible variation for risk of CSM over time. Specifically, MCR models were fitted in the overall population at baseline (time zero) and, subsequently, 8 separate additional MCR models were fitted in patients who survived 1, 2, 3, 4, 5, 6, 7, and 8 years after surgery. The variables of interest were $T$ and $\mathrm{N}$ stage, Fuhrman grade, and histologic subtype. Additional adjustment variables consisted of age, sex, ethnicity, and type of surgery. Finally, we tested for interaction between Fuhrman grade/histologic subtype and primary risk factor, namely $\mathrm{T}$ and $\mathrm{N}$ stages.

All statistical tests were 2-sided with a level of significance set at $P<.05$. Analyses were performed using $\mathrm{R}$ version 3.4.1 (R Foundation for Statistical Computing).

\section{Results}

General Characteristics of the Study Population

From 2001 through 2015, 77,892 patients with nmRCC were identified, $26,562(34.1 \%)$ of whom were treated with partial nephrectomy and 51,330 (65.9\%) with radical nephrectomy. Stage distribution was as follows: 35,966 patients $(46.2 \%)$ with pTlaN0; $18,858(24.2 \%)$ with pT1bN0; 5,977 (7.7\%) with pT2aN0; 2,511 (3.2\%) with pT2bN0; 11,839 (15.2\%) with pT3aN0; 1,037 (1.3\%) pT3b-cN0; 402 (0.5\%) with pT4N0; and 1,302 (1.7\%) with pTanyN1 (Table 1).

\section{Conditional 5-Year CSM Analyses}

The baseline (time zero) 5-year CSM-free rate in pTlaN0 cohort (35,966 patients) was $98.2 \%$. Notably, a stable $2 \%$ 5 -year CSM risk persisted up to 10 years of event-free

\section{Table 1. Patient Characteristics}

\begin{tabular}{|c|c|}
\hline Variable & n (\%) \\
\hline Median age (IQR), y & $61(53-70)$ \\
\hline \multicolumn{2}{|l|}{ Ethnicity } \\
\hline White & $64,190(82.4)$ \\
\hline African American & $8,360(10.7)$ \\
\hline Asian & $4,144(5.3)$ \\
\hline Other & $1,198(1.5)$ \\
\hline \multicolumn{2}{|l|}{ Sex } \\
\hline Male & $49,409(63.4)$ \\
\hline Female & $28,483(36.6)$ \\
\hline \multicolumn{2}{|l|}{ Marital status } \\
\hline Married & $49,364(63.4)$ \\
\hline Never married & $11,064(14.2)$ \\
\hline Previously married & 13,933 (17.9) \\
\hline Unknown & $3,531(4.5)$ \\
\hline \multicolumn{2}{|l|}{ Year of diagnosis } \\
\hline 2001-2005 & $14,298(18.4)$ \\
\hline 2006-2010 & $29,135(37.4)$ \\
\hline 2011-2015 & $34,459(44.2)$ \\
\hline \multicolumn{2}{|l|}{ Type of surgery } \\
\hline Partial nephrectomy & $26,562(34.1)$ \\
\hline Radical nephrectomy & $51,330(65.9)$ \\
\hline \multicolumn{2}{|l|}{ Tumor stage } \\
\hline pT1aNo & $35,966(46.2)$ \\
\hline pT1bN0 & $18,858(24.2)$ \\
\hline pT2aNo & $5,977(7.7)$ \\
\hline pT2bNo & $2,511(3.2)$ \\
\hline pT3aNO & $11,839(15.2)$ \\
\hline pT3b-cNO & $1,037(1.3)$ \\
\hline pT4N0 & $402(0.5)$ \\
\hline pTanyN1 & $1,302(1.7)$ \\
\hline \multicolumn{2}{|l|}{ Fuhrman grade } \\
\hline 1 & $9,824(12.6)$ \\
\hline 2 & $42,714(54.8)$ \\
\hline 3 & $21,371(27.4)$ \\
\hline 4 & $3,983(5.1)$ \\
\hline \multicolumn{2}{|l|}{ Histology } \\
\hline Clear-cell & $60,398(77.5)$ \\
\hline Papillary & $12,200(15.7)$ \\
\hline Chromophobe & $4,551(5.8)$ \\
\hline Sarcomatoid & $743(1.0)$ \\
\hline
\end{tabular}

Abbreviation: IQR, interquartile range.

follow-up. In pT1bN0 cohort (18,858 patients), a 5\% 5 -year CSM risk remained stable from beginning up to 10 years of event-free follow-up (Figure 1, Table 2). 
A

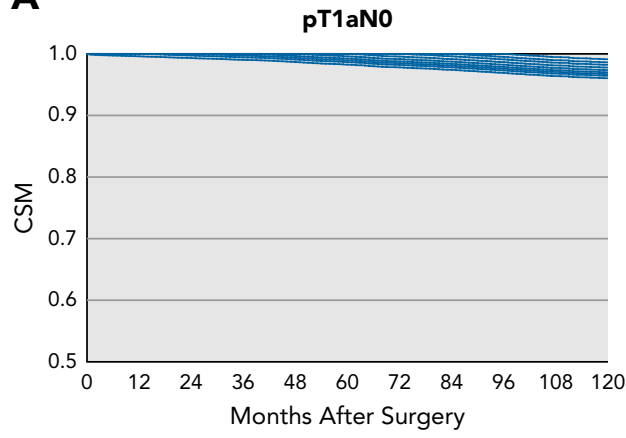

C

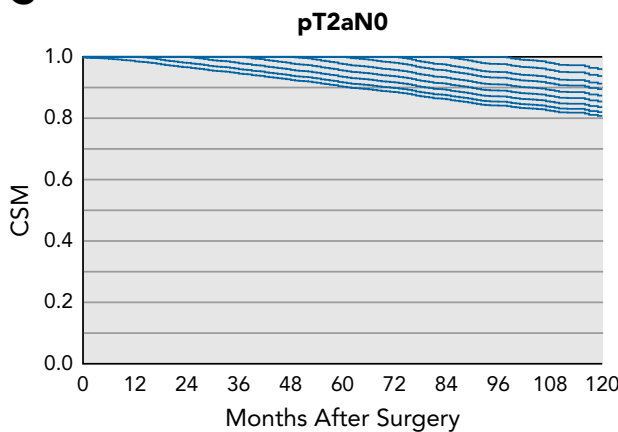

E

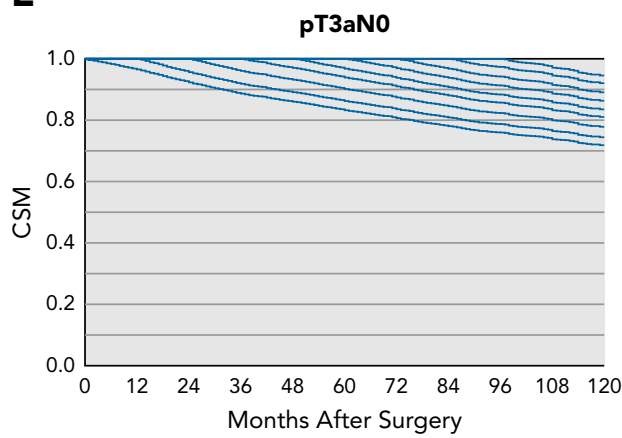

G

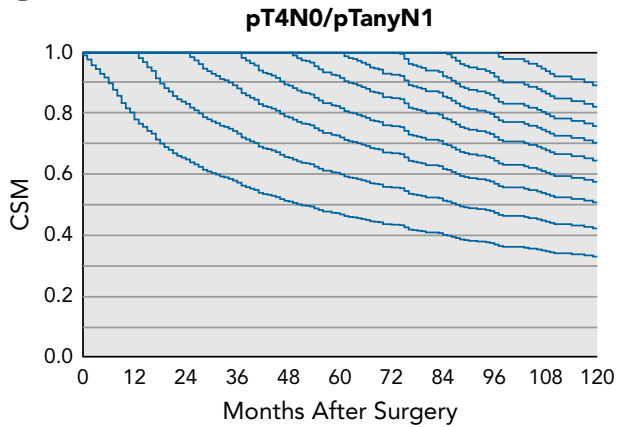

B

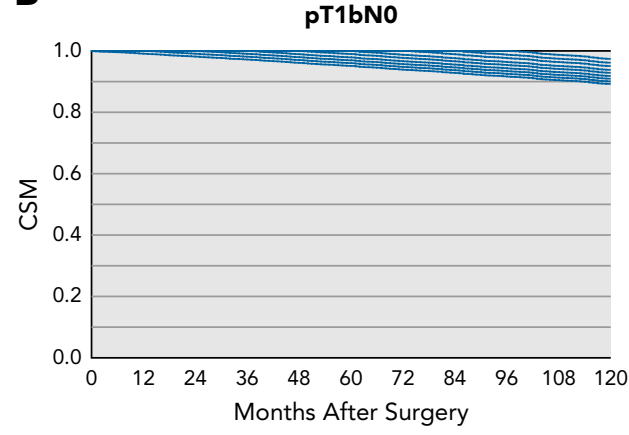

D

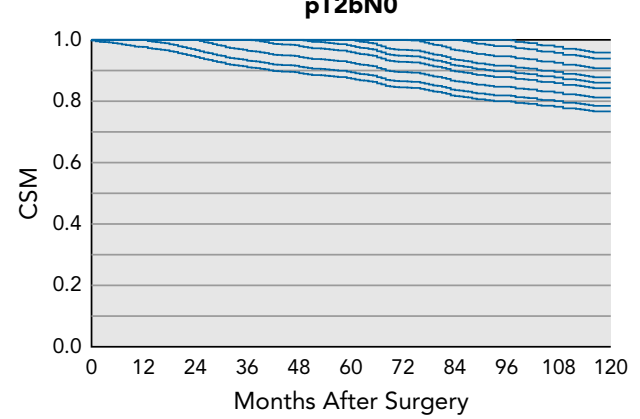

F

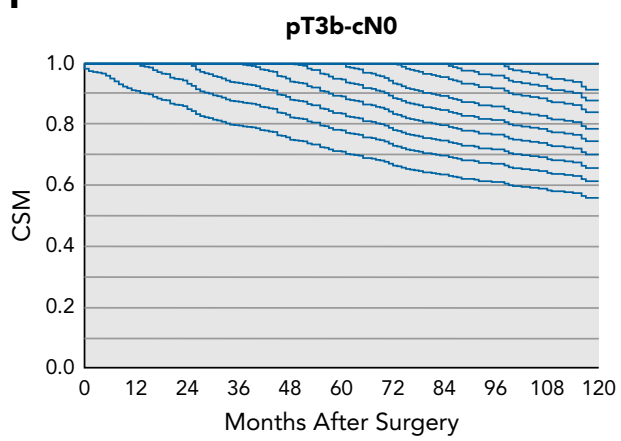

Figure 1. Conditional CSM-free survival rates after nephrectomy for patients with (A) pT1aN0, (B) pT1bN0, (C) pT2aN0, (D) pT2bN0, (E) pT3aN0, (F) pT3b-cN0, and (G) pT4N0/pTanyN1 nonmetastatic renal cell carcinoma.

Abbreviation: CSM, cancer-specific mortality.

In the pT2aN0 cohort $(n=5,977)$, the 5 -year CSMfree survival probabilities declined from $90.3 \%$ at baseline to $89.5 \%$ and $88.7 \%$, respectively, at 60 and 120 months of event-free follow-up. Conversely, in the
pT2bN0 cohort $(n=2,511)$, the 5-year CSM-free survival probabilities increased from $87.3 \%$ at baseline to $91.1 \%$ at 96 months of event-free follow-up (Figure 1, Table 2). 


\begin{tabular}{|c|c|c|c|c|c|c|c|c|c|}
\hline \multirow[b]{2}{*}{ Cohort } & \multicolumn{9}{|c|}{ Months Survived } \\
\hline & 0 & 12 & 24 & 36 & 48 & 60 & 72 & 96 & 120 \\
\hline \multicolumn{10}{|l|}{ pT1aNo } \\
\hline At risk, $\mathrm{n}^{\mathrm{a}}$ & 35,966 & 31,103 & 27,200 & 23,439 & 19,948 & 16,691 & 13,765 & 8,199 & 4,125 \\
\hline Survival gain, \%c & - & +0.0 & -0.1 & -0.3 & -0.5 & -0.5 & -0.8 & -1.0 & -0.2 \\
\hline \multicolumn{10}{|l|}{ pT1bN0 } \\
\hline At risk, $\mathrm{n}^{\mathrm{a}}$ & 18,858 & 16,257 & 14,081 & 12,076 & 10,204 & 8,559 & 6,897 & 4,195 & 2,111 \\
\hline Events, $\mathrm{n}^{\mathrm{b}}$ & 20 & 144 & 144 & 128 & 123 & 97 & 101 & 62 & 83 \\
\hline At risk, $\mathrm{n}^{\mathrm{a}}$ & 5,977 & 5,144 & 4,503 & 3,918 & 3,306 & 2,766 & 2,265 & 1,371 & 716 \\
\hline Events, $n^{b}$ & 15 & 68 & 95 & 90 & 79 & 71 & 49 & 38 & 42 \\
\hline Conditional 5-y CSM-free survival, \% ${ }^{\mathrm{a}}$ & 90.3 & 89.9 & 89.4 & 89.1 & 89.3 & 89.5 & 89.1 & 88.7 & 88.7 \\
\hline Survival gain, $\%^{c}$ & - & -0.4 & -0.9 & -1.2 & -1.0 & -0.8 & -1.2 & -1.6 & -1.6 \\
\hline \multicolumn{10}{|l|}{ pT2bNO } \\
\hline At risk, $\mathrm{n}^{\mathrm{a}}$ & 2,511 & 2,143 & 1,839 & 1,581 & 1,366 & 1,148 & 934 & 598 & - \\
\hline Events, $\mathrm{n}^{\mathrm{b}}$ & 6 & 48 & 67 & 62 & 31 & 25 & 34 & 14 & - \\
\hline Conditional 5-y CSM-free survival, \% ${ }^{\mathrm{a}}$ & 87.3 & 86.5 & 86.7 & 87.9 & 87.7 & 87.8 & 87.7 & 91.1 & - \\
\hline Survival gain, \%c & - & -0.8 & -0.6 & +0.6 & +0.4 & +0.5 & +0.4 & +3.8 & \\
\hline At risk, $\mathrm{n}^{\mathrm{a}}$ & 1,037 & 824 & 697 & 561 & 462 & 386 & 311 & 234 & - \\
\hline Events, $\mathrm{n}^{\mathrm{b}}$ & 17 & 70 & 51 & 41 & 30 & 22 & 23 & 10 & - \\
\hline Conditional 5-y CSM-free survival, \% ${ }^{\mathrm{a}}$ & 71.1 & 73.7 & 74.7 & 76.7 & 77.8 & 78.1 & 80.5 & 79.4 & - \\
\hline Survival gain, \%c & - & +2.6 & +3.6 & +5.6 & +6.7 & +7.0 & +9.4 & +8.3 & \\
\hline \multicolumn{10}{|l|}{ pT4N0/pTanyN1 } \\
\hline At risk, $\mathrm{n}^{\mathrm{a}}$ & 1,704 & 1,117 & 771 & 596 & 448 & 345 & 263 & 150 & - \\
\hline Events, $n^{b}$ & 15 & 319 & 169 & 84 & 59 & 34 & 23 & 14 & - \\
\hline Conditional 5-y CSM-free survival, \% ${ }^{a}$ & 46.8 & 55.8 & 62.7 & 65.3 & 68.5 & 70.4 & 69.4 & 77.9 & - \\
\hline Survival gain, $\%^{c}$ & - & +9.0 & +15.9 & +18.5 & +21.7 & +23.6 & +22.6 & +31.1 & \\
\hline
\end{tabular}

Abbreviation: CSM, cancer-specific mortality.

${ }^{a}$ At the beginning of the interval.

${ }^{\mathrm{b}} \mathrm{At}$ the end of the interval.

'Relative to baseline.

In the pT3aN0 cohort $(n=11,839)$, the 5 -year CSMfree survival probabilities increased from $83.2 \%$ at baseline to $89.6 \%$ at 120 months of event-free follow-up. Similarly, in the pT3b-cN0 cohort $(n=1,037)$, the 5 -year CSM-free survival probabilities increased from $71.1 \%$ at baseline to $79.4 \%$ at 96 months of event-free follow-up. Finally, in the pT4N0/pTanyN1 cohort $(n=1,704)$, the 5 -year CSM-free survival probabilities increased from $46.8 \%$ at baseline to $77.9 \%$ at 96 months of event-free follow-up (Figure 1, Table 2). 
Attrition due to mortality was lowest in patients with pTlaN0 disease (Table 2), as evidenced by conditional interval-specific CSM-free rates that ranged from $98.2 \%$ to $98.0 \%$, respectively, at baseline and 10 years of eventfree follow-up. Conversely, attrition due to mortality was highest in patients with pT4N0/pTanyN1 disease, as evidenced by conditional interval-specific CSM-free rates that ranged from $46.8 \%$ to $77.9 \%$, respectively, at baseline and 8 years of event-free follow-up.

\section{MCR Models Predicting CSM}

In MCR models predicting CSM (Figure 2, Table 3), tumor stage, Fuhrman grade, and histologic subtype were independent predictors of CSM risk. However, no interactions were identified. Regarding the effect of stage, relative to the referent pT1N0 stage, the magnitude of decreases in CSM hazard was virtually stable for pT2N0 (hazard ratio [HR], 3.3 at baseline to 2.6 at 96 months of event-free follow-up), intermediate for pT3N0 (HR, from 4.4 to 2.8, respectively), and highest for patients with pT4N0/pTanyN1 disease (HR, from 15.2 to 6.7 , respectively).

For Fuhrman grade, relative to the referent grade 1, the CSM hazard was no more significant after 48 months of EFS for grade 2. Conversely, a decrease in the magnitude of CSM hazard risk was recorded for both grade 3 (HR, from 2.1 at baseline to 1.5 at 96 months of event-free follow-up) and grade 4 (HR, from 3.8 to 2.0, respectively).

A

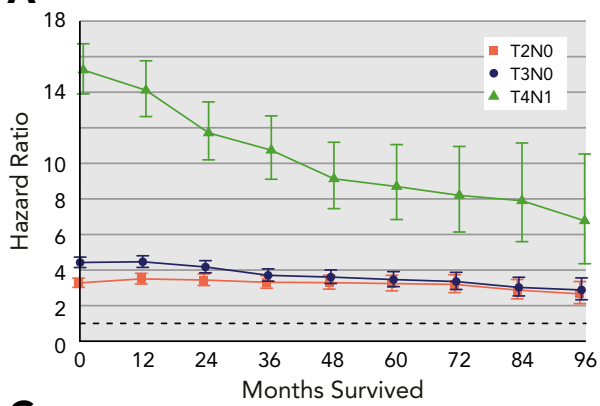

C

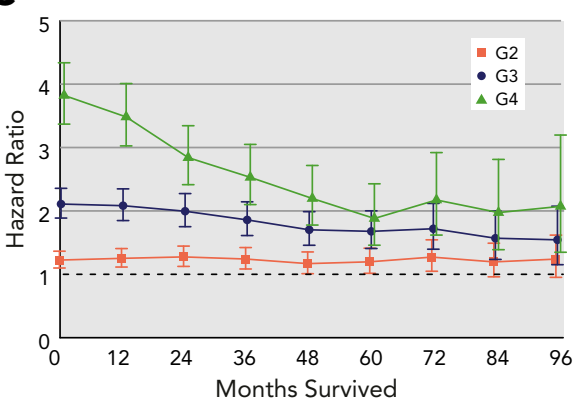

Lastly, for histologic subtype, relative to the referent clear-cell histology, both chromophobe (HR, 0.4 at baseline to 0.5 at 96 months of event-free follow-up) and papillary histology (HR, from 0.8 to 0.6 , respectively) were stable. Conversely, the CSM hazard was no more significant after 24 months of EFS for sarcomatoid.

\section{Discussion}

CS represents an important entity as evidenced by previous reports in both genitourinary ${ }^{6-8}$ and other primaries. ${ }^{1,9,10}$ This notion was pioneered by Skuladottir and Olsen ${ }^{1}$ in patients with lung cancer in 2003. Despite the importance of CS and its universal use in patient counseling, only 5 formal studies specifically quantified CS in patients with RCC., ${ }^{2,3,11-13}$ Of these, 2 exclusively focused on metastatic RCC, ${ }^{11,13}$ showing that despite the aggressiveness and incurable nature of the disease, CS represented an important consideration in these patients, as evidenced by statistically significant and clinically meaningful improvements in $\mathrm{CSM}^{11}$ and overall mortality, ${ }^{13}$ provided there was EFS after diagnosis and/or initiation of systemic therapy.

Of the remaining 3 reports, only $2^{2,3}$ addressed cancerspecific CS in nmRCC. Unfortunately, neither of these 2 studies provided stratified estimates of conditional CSM-free survival according to $\mathrm{T}$ and $\mathrm{N}$ stage and substage groupings. Substage stratification is particularly

\section{B}

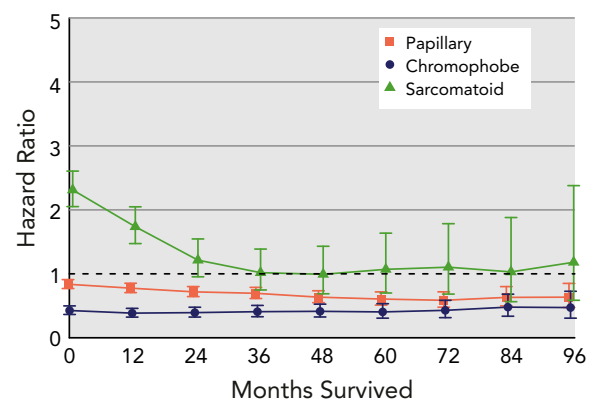

Figure 2. Multivariable Cox proportional hazards regression predicting CSM after nephrectomy according to (A) tumor stage (referent: $\mathrm{pT} 1 \mathrm{~N} 0$ ), (B) histologic subtype (referent: clear cell), and (C) Fuhrman grade (referent: G1). Additional adjustment variables consisted of age, sex, ethnicity, and type of surgery.

Abbreviations: CSM, cancer-specific mortality; G, Fuhrman grade. 


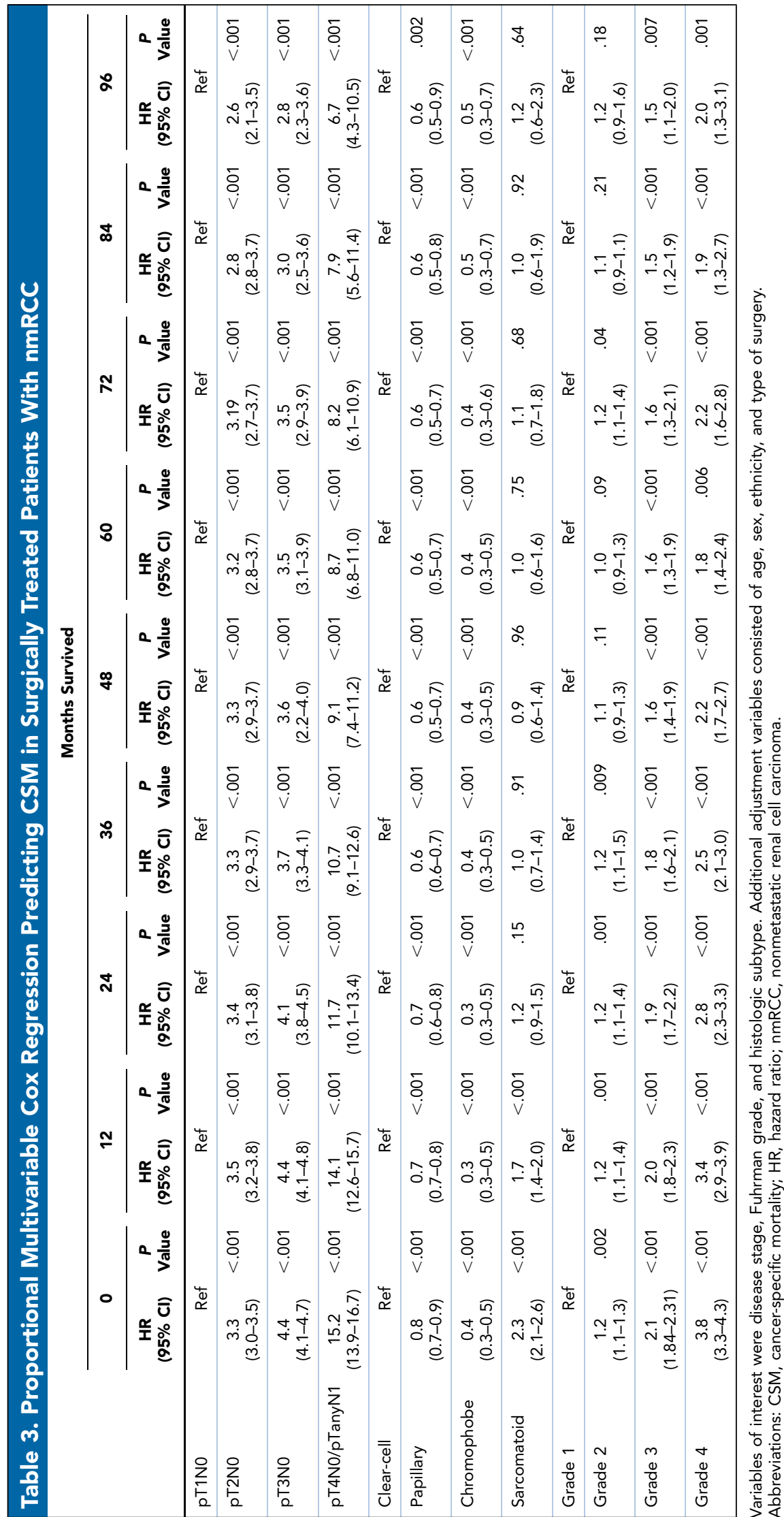


important in contemporary urologic practice, because most patients with newly diagnosed nmRCC have been shown to harbor Tla tumors. ${ }^{14}$ Moreover, patients with favorable disease characteristics, such as those with Tla or even Tlb tumors, may have a dramatically different prognosis from their counterparts with more advanced disease stage. Nonetheless, equally important prognostic difference in CS may also exist between substages of T2 or even T3 nmRCC.

Based on these hypotheses, we performed a detailed analysis of CS within a large contemporary populationbased cohort of nmRCC. Our analysis provided the first stratified CSM estimates according to T substages and is summarized as follows.

First, the largest proportion of patients harbor pTlaNo $(n=35,966 ; 46.2 \%)$ and pT1bNo $(n=18,858$; $24.2 \%$ ) substages, followed by pT3aNo (15.2\%), pT2aN0 (7.7\%), pT2bN0 (3.2\%), pTanyN1 (1.7\%), pT3b-cN0 (1.3\%), and pT4N0 $(0.5 \%)$ stage and substages groupings. Consequently, from a clinical and epidemiologic perspective, stratification according to $\mathrm{T}$ and $\mathrm{N}$ stage and substage groupings is particularly important in the most frequent subgroupings, namely pTlaN0 and pTlbN0. Nonetheless, from the perspective of individual patient counseling, stratification of CS based on prognosis is equally important in those with more advanced disease, such as pT3N0, pT4N0, and even pTanyN1, based on substantially worse prognoses for these individuals. This point validates the need for substage stratification of CS.

Second, CS estimates after 5-year EFS ranged from a maximum of $97.7 \%$ to a minimum $70.4 \%$ for patients with pTlaNo and pT4N0/pTanyN1 disease, respectively. These results illustrate very important heterogeneity in the prognosis of surgically treated nmRCC according to stage and substage groupings, and again validate the rationale of our study, which reports stage and substage grouping stratification of CS estimates.

Third, patients with pTlaNo disease show CS estimates that are virtually unchanged during a 10-year event-free follow-up $(98.2 \%$ after 1 year vs $98.0 \%$ after 10 years). From a clinical perspective, this implies that ongoing follow-up of patients with pT1aNo disease is based on a $2 \%$ CSM risk, indicating that 50 individuals should be investigated in an ongoing fashion to identify 1 that will eventually die. Despite these low CSM rates, current European guidelines ${ }^{15}$ do not define any particular time point at which follow-up should be interrupted. Similarly, the most recent version of the NCCN Clinical Practice Guidelines in Oncology (NCCN Guidelines) for Kidney Cancer ${ }^{16}$ at the time of writing did not recommend a specific cutoff with respect to duration of follow-up. Instead, follow-up should be tailored based on patient and tumor characteristics. We identified virtually the same phenomenon for patients with pTlbN0 disease, with the exception of CSM risk, which was recorded at 5\%.
Fourth, a gradual decrease in CSM rates after periods of event-free follow-up was recorded in subgroupings of patients with pT2aN0, pT2bN0, pT3aN0, and pT3b-cN0 disease. Conversely, a more prominent decrease in CSM rates after shorter periods of event-free follow-up was recorded for patients with pT4N0/pTanyN1 disease (from $46.8 \%$ after 1 year vs $77.9 \%$ after 8 years of event-free follow-up). Consequently, patients with the most aggressive $\mathrm{T}$ - and $\mathrm{N}$-stage subgroupings benefit the most from event-free follow-up. Conversely, patients with the most favorable T substages (pTlaN0 and pTlbN0) do not benefit from a decreasing CSM risk event after a lengthy period of event-free follow-up.

Fifth, we also identified important differences in CSM during the intervals of observation that distinguish patients with specific stages and substages. Highest CSM was recorded in patients with pT4N0/pTanyN1 disease, as evidenced by conditional interval-specific CSM-free rates as low as $46.8 \%$ initially that eventually improved to $77.9 \%$ after 8 years of event-free follow-up. Conversely, the lowest CSM was recorded in patients with pTlaN0 disease, as evidenced by conditional intervalspecific CSM-free rates as high as $98.2 \%$ initially that remained stable at $98.0 \%$ after 10 years of event-free follow-up. These observations validate again the extreme heterogeneity of prognoses for patients with nmRCC. ${ }^{17}$

Sixth, very similar findings were recorded after stratification according to Fuhrman grade and histologic subtype. Based on the absence of statistically significant interactions between $\mathrm{T}$ stage and its substages and either Fuhrman grade or histologic subtype, we abstained from additional stratification schemes according to those variables.

Several take-home messages warrant consideration. First, patients with pTlaN0 and pTlbN0 disease showed the lowest 5 -year CSM rates, at $2 \%$ and $5 \%$, respectively, and these did not change with the duration of event-free follow-up that ranged from 1 to 10 years. Conversely, for stages pT2aN0 to pT4N0/pTanyN1, event-free follow-up resulted in higher CSM-free rates. Second, the magnitude of CSM decrease was most significant in patients with pT4N0/pTanyN1 disease and least significant in patients with pT2aN0 disease. Third, in patients with pT2aN0 to pT4N0/pTanyN1 disease, the absolute decrease in CSM occurred after the shorter event-free follow-up interval in those with the most advanced stages, whereas it occurred over the longest interval in those with a less-advanced stage (pT2aN0). Lastly, the lowest substages (pT1aN0 and pTlbN0) represent the largest subgroups with the least attrition due to mortality over time. However, the highest $\mathrm{T}$ and $\mathrm{N}$ stage and substage groupings represent the smallest subgroup; nonetheless, the amount of attrition due to mortality is the highest. In summary, our findings should be considered in determining the duration of substage-specific follow-up for surgically treated patients 
with nmRCC disease. To the best of our knowledge, CS substage-specific follow-up protocols have not been adopted by either European Association of Urology ${ }^{15}$ or $\mathrm{NCCN}^{16}$ guidelines, especially for patients with pTlaNo and pTlbN0 disease.

Despite the strengths of this study, important limitations need to be acknowledged. First, our data represents a retrospective analysis with high potential for selection biases. Second, our data that relied on CSM as an end point do not represent an ideal source for defining a specific surveillance protocol. Instead, recurrence and metastatic progression data, which are not available within the SEER database, should be used for that purpose. Nonetheless, our findings represent the most informative method of providing CS probabilities in surgically treated patients with nmRCC. Therefore, although our study could not rely on either recurrence or progression data, clinicians should be aware of changes in CS according to specific substages and should ideally incorporate them in decision-making. Third, no information about comorbidities is available in the SEER database. Fourth, because of the SEER coding system, we could not account for reclassification of AJCC staging from 2010 onward; we relied on the 6th edition of the AJCC Cancer Staging Manual for patients diagnosed from 2001 through 2009 according to the SEER database, and on the 7th edition for those diagnosed from 2010 onward. Specifically, adrenal invasion is classified as T3a in the 6th edition versus T4 in the 7th edition. Finally, the amount of detail is limited relative to smaller institutional studies. For example, laboratory values and performance status could not be assessed. However, these limitations apply to all population-based analyses that were based on SEER, the National Cancer Database, or other nationbased data repositories.

\section{Conclusions}

CSM risk showed important variability across the range of examined stage categories. For example, CSM risk of patients with pTlaNo disease remained stable, even after 10-year event-free follow-up. Conversely, prognosis for patients with stage pT4N0/pTanyN1 disease may drastically improve in proportion to event-free follow-up duration. Our observations have critical importance for physicians regarding patient follow-up and counseling.

Submitted May 23, 2019; accepted for publication August 28, 2019.

Author contributions: Study concept and design: Palumbo, Karakiewicz. Data acquisition: Palumbo, Mistretta, Knipper, Pecoraro. Data analysis and interpretation: Palumbo, Mistretta, Knipper, Pecoraro, Karakiewicz. Statistical analysis: Palumbo, Tian. Drafting of manuscript: Palumbo, Karakiewicz. Critical revision for important intellectual content: Shariat, Saad, Simeone, Briganti, Antonelli. Final approval of manuscript: All authors. Supervision: Shariat, Saad, Simeone, Briganti, Antonelli, Karakiewicz.

Disclosures: The authors have disclosed that they have not received any financial consideration from any person or organization to support the preparation, analysis, results, or discussion of this article.

Correspondence: Carlotta Palumbo, MD, Urology Unit, ASST Spedali Civili of Brescia, Department of Medical and Surgical Specialties, Radiological Science and Public Health, University of Brescia, Piazzale Spedali Civili 1 25123, Brescia, Italy. Email: palumbo.carlotta@gmail.com

\section{References}

1. Skuladottir $\mathrm{H}$, Olsen $\mathrm{JH}$. Conditional survival of patients with the four major histologic subgroups of lung cancer in Denmark. J Clin Oncol 2003; 21:3035-3040.

2. Bianchi M, Becker A, Hansen J, et al. Conditional survival after nephrectomy for renal cell carcinoma (RCC): changes in future survival probability over time. BJU Int 2013;111:E283-289.

3. Karakiewicz Pl, Suardi N, Capitanio U, et al. Conditional survival predictions after nephrectomy for renal cell carcinoma. J Urol 2009;182:2607-2612.

4. National Cancer Institute. About the SEER Program. Available at: https:// seer.cancer.gov/about/. Accessed August 1, 2019.

5. Hieke S, Kleber M, König C, et al. Conditional survival: a useful concept to provide information on how prognosis evolves over time. Clin Cancer Res 2015;21:1530-1536.

6. Stephenson AJ, Scardino PT, Eastham JA, et al. Postoperative nomogram predicting the 10-year probability of prostate cancer recurrence after radical prostatectomy. J Clin Oncol 2005;23:7005-7012.

7. Sun M, Abdollah F, Bianchi M, et al. Conditional survival of patients with urothelial carcinoma of the urinary bladder treated with radical cystectomy. Eur J Cancer 2012;48:1503-1511.

8. Ploussard G, Shariat SF, Dragomir A, et al. Conditional survival after radical cystectomy for bladder cancer: evidence for a patient changing risk profile over time. Eur Urol 2014;66:361-370.

9. Henson DE, Ries LA, Carriaga MT. Conditional survival of 56,268 patients with breast cancer. Cancer 1995;76:237-242.

10. Groth SS, Rueth NM, Hodges JS, et al. Conditional cancer-specific versus cardiovascular-specific survival after lobectomy for stage I non-small cell lung cancer. Ann Thorac Surg 2010;90:375-382.
11. Harshman LC, Xie W, Bjarnason GA, et al. Conditional survival of patients with metastatic renal-cell carcinoma treated with VEGFtargeted therapy: a population-based study. Lancet Oncol 2012;13: 927-935.

12. Abdollah F, Suardi N, Capitanio U, et al. The key role of time in predicting progression-free survival in patients with renal cell carcinoma treated with partial or radical nephrectomy: conditional survival analysis. Urol Oncol 2014;32:43.e9-43.e16.

13. Kang M, Park JY, Jeong CW, et al. Changeable conditional survival rates and associated prognosticators in patients with metastatic renal cell carcinoma receiving first line targeted therapy. J Urol 2018; 200:989-995.

14. Turner RM II, Morgan TM, Jacobs BL. Epidemiology of the small renal mass and the treatment disconnect phenomenon. Urol Clin North Am 2017;44:147-154.

15. Ljungberg B, Albiges L, Bensalah K, et al. European Association of Urology Guidelines, 2018 edition. Presented at the 32nd Annual EAU Congress; March 16-20, 2018; Copenhagen, Denmark.

16. Motzer RJ, Jonasch E, Agarwal N. NCCN Clinical Practice Guidelines in Oncology: Kidney Cancer. Version 1.2019. Accessed August 1, 2019. To view the most recent version, visit NCCN.org.

17. Brookman-May S, May M, Shariat SF, et al. Features associated with recurrence beyond 5 years after nephrectomy and nephron-sparing surgery for renal cell carcinoma: development and internal validation of a risk model (PRELANE score) to predict late recurrence based on a large multicenter database (CORONA/SATURN Project). Eur Urol 2013;64: 472-477. 